\title{
Quel avenir pour la coloproctologie dans la prochaine décennie ?
}

\author{
G. Meurette \\ (C) Springer-Verlag France 2010
}

Une nouvelle décennie s'ouvre avec la nouvelle année, la coloproctologie française doit prétendre à un avenir qui s'inscrit sous le signe du progrès, de l'innovation et de l'unité !

Drôle de spécialité naissant du mariage entre des gastroentérologues proctologues, qui progressivement revendiquent une spécialité authentique au sein de l'hépatogastroentérologie, emmenant dans leur sillage les endoscopistes interventionnels et les chirurgiens viscéraux résolument tournés vers la « colorectale », la coloproctologie à la française fait lentement son chemin vers l'unité. Finalement, les efforts consentis par toutes les parties ont forgé son identité et permis de lui dessiner un visage dynamique. Il faut reconnaître que la richesse des pathologies, avec la cancérologie colorectale et anale, les maladies inflammatoires, la proctologie médicochirurgicale, la statique pelvienne, l'incontinence fécale, etc., participe activement à développer la spécialité, tant les avancées récentes lui confèrent un caractère jeune et enthousiaste dans ses différents champs d'exercice !

Les perspectives de développement considérables qui s'offrent à nous pour les dix ans à venir devraient faire pâlir de jalousie nos collègues spécialistes en pathologie hépatobiliaire et susciter l'enthousiasme et la vocation des plus jeunes internes en médecine et en chirurgie.

Prenons quelques exemples parmi les plus frappants :

- dans le domaine de l'endoscopie, tout d'abord, les innovations technologiques font reculer davantage les limites des résections chirurgicales de lésions coliques ou rectales par dissection sous-muqueuse dans des conditions de visibilité et de sécurité toujours croissantes. Il devient possible d'entreprendre l'exérèse de nappes villeuses étendues sans fragmenter les pièces et épargner aux patients des colectomies sources de morbidité significative ; de quoi appréhender l'arrivée de la coloscopie virtuelle avec de sérieux arguments de pertinence à opposer! Par ailleurs, les prises en charge combinées médicochirurgicales, sous

\footnotetext{
G. Meurette $(\bowtie)$

Institut des maladies de l'appareil digestif,

CHU de Nantes, 5, allée de l'Île-Gloriette,

F-44093 Nantes cedex 01, France

e-mail : guillaume.meurette@wanadoo.fr
}

contrôle laparoscopique peuvent ainsi s'envisager dans des conditions carcinologiques raisonnables aussi. Dans le domaine diagnostique, la microendoscopie confocale [1] se développe et dépasse désormais le domaine de la médecine expérimentale, elle s'apprête à faire partie de l'investigation clinique pour le diagnostic histologique in situ des lésions coliques ;

- la microchirurgie par voie transanale [2] (ou TEM des Anglo-Saxons, pour transanal endoscopic microsurgery) gagne également du terrain et des indications dans le domaine de la pathologie tumorale rectale. Les arguments dans la littérature se multiplient en faveur de cette approche mini-invasive. On sait aujourd'hui que la résection rectale, aussi réglée soit-elle, expose le patient à des complications postopératoires significatives et à des résultats fonctionnels incertains. Une population vieillissant et un diagnostic porté plus précocement font que de petites tumeurs rectales bénignes ou dégénérées (à un stade précoce) sont de plus en plus fréquemment dépistées dans notre pratique quotidienne et incitent à envisager ces techniques mini-invasives ;

- la laparoscopie robot-assistée [3] apporte également son lot d'innovation technologique aux chirurgiens colorectaux, 30 ans après la naissance de la cœlioscopie et ses débuts laborieux que nous avons tous encore en mémoire. La dissection du mésorectum sous contrôle robot devrait donner au chirurgien un confort visuel qui augmentera la qualité de l'exérèse. Certes, les résultats sont encore incertains, car trop récents, et il faut rester prudent avant de diffuser cette approche, nul ne peut cependant ignorer ce progrès dans l'innovation optique, ergonomique des instruments confectionnés pour être utilisés sous robot, et le confort donné à l'opérateur en lui évitant crampes et lombalgies...;

- dans le domaine de la proctologie, la chirurgie hémorroïdaire voit son arsenal thérapeutique évoluer avec les ligatures des artères hémorroïdales sous contrôle doppler [4], le développement de pinces mécaniques à ergonomie croissante, les dispositifs hémostatiques plus performants. Toutes ces approches permettront d'offrir aux patients un confort postopératoire, supérieur à ce que procure la chirurgie conventionnelle, et un risque moindre de complications. Peut-être peut-on espérer, enfin, changer la mauvaise image de la chirurgie des hémorroïdes dans la population ? 
Bien sûr, si l'enthousiasme nous pousse vers le développement de notre spécialité jeune et s'ouvre au progrès technologique, ces innovations ont un coût important que nous devons mesurer. La bonne intelligence sera d'être capable de pondérer l'élan vers le progrès souvent le souhait des plus jeunes, passionnés dans un monde où « technologie » est synonyme de modernité et de confort, sans pour autant freiner ou retarder un inéluctable rendez-vous avec l'histoire de la coloproctologie. Tirons les leçons du passé et donnons-nous les moyens de faire avancer la coloproctologie à la française, au regard des autorités de tutelle et autres organismes payeurs, faute de quoi, d'autres en profiteront, et nous n'aurons que nos yeux pour pleurer, et des livres d'histoires pour nous rappeler le temps glorieux de grands noms de médecins français à la pointe du progrès... du siècle dernier. Nous serions démunis, face à l'expansion de la coloproctologie européenne, américaine et asiatique, priant que l'industrie qui est actrice incontournable de cette innovation accepte de traduire les manuels d'utilisation de leur matériel en français !

$\mathrm{Au}$ lieu de cela, débutons la prochaine décennie sereinement, profitant de l'expérience des plus anciens et de l'enthou- siasme des plus jeunes : qu'ils montrent la direction à suivre, éveillent les vocations coloproctologiques chez les étudiants, canalisent les pressions médicoéconomiques, orientent les pistes à développer pour mieux soigner nos patients, fassent entendre la voix des coloproctologues français sur la scène européenne... Soyons capables d'avancer sous le signe du progrès, de l'innovation et de l'unité.

\section{Références}

1. De Palma GD (2009) Confocal laser endomicroscopy in the "in vivo" histological diagnosis of the gastrointestinal tract. World J Gastroenterol 15(46):5770-5

2. Allaix ME, Arezzo A, Caldart M, et al (2009) Transanal endoscopic microsurgery for rectal neoplasms: experience of 300 consecutive cases. Dis Colon Rectum 52(11):1831-6

3. Luca F, Cenciarelli S, Valvo M, et al (2009) Full robotic left colon and rectal cancer resection: technique and early outcome. Ann Surg Oncol 16(5):1274-8

4. Giordano P, Overton J, Madeddu F, et al (2009) Transanal hemorrhoidal dearterialization: a systematic review. Dis Colon Rectum 52(9):1665-71 